INFLAMMATORY BOWEL DISEASE

\title{
Clinical and subclinical intestinal inflammation assessed by the mucosal patch technique: studies of mucosal neutrophil and eosinophil activation in inflammatory bowel diseases and irritable bowel syndrome
}

\author{
G Kristjánsson, P Venge, A Wanders, L Lööf, R Hällgren
}

Gut 2004;53:1806-1812. doi: 10.1136/gut.2003.036418

Background and aims: There is a clear need for a rapid, simple, safe, and sensitive method of determining the type and intensity of inflammation in the gut mucosa in clinical practice. In this study, we have evaluated the potential of a new method, the mucosal patch technique, in patients with and without apparent gut inflammation, as assessed by conventional diagnostic procedures.

See end of article for authors' affiliations

Correspondence to: Dr G Kristiánsson, Department of Medical Sciences, University of Uppsala, University Hospital of Uppsala, 75185 Uppsala, Sweden; gudjon.kristjansson@ medsci.uu.se

Revised version received 28 April 2004

Accepted for publication 11 May 2004
Subjects and methods: The technique tested is based on the idea that inflammatory mediators released from the rectal mucosa can be absorbed by and then extracted from cellulose patches brought into contact with the mucosa by use of an instrument with an inflatable balloon. Measurements were performed in healthy controls $(n=16)$ and in patients with active $(n=19)$ and inactive ulcerative colitis $(U C, n=8)$, collagen colitis (CC, $n=12)$, coeliac disease $(n=13)$, and irritable bowel syndrome (IBS, $n=13)$.

Results: Inflammatory mediators from neutrophils (myeloperoxidase (MPO)) and eosinophils (eosinophil cationic protein (ECP)) were increased on average 300- and 10-fold, respectively, in patients with active UC compared with healthy controls and were correlated with the endoscopic score. Patients with inactive UC, CC, coeliac disease, and IBS exhibited no endoscopic signs of inflammation. These patient groups had significantly lower levels of MPO and ECP than the active UC group but showed on average a four- to sevenfold increase in MPO compared with healthy controls.

Conclusion: The mucosal patch technique was well tolerated by patients and easily applied by the investigator. Pronounced neutrophil and eosinophil involvement in UC was demonstrated. With the high sensitivity of the technique, low degree mucosal neutrophil activation could also be quantified in patients with CC and UC in clinical remission. The finding of increased neutrophil involvement in patients with IBS contributes to the pathophysiological ideas of this disease.

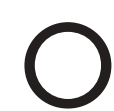
ur knowledge of the events in the wall of the colorectum in various diseases has long been derived from morphological and immunohistochemical studies of biopsy and surgical specimens from affected gut areas. $^{1-3}$ In the last two to three decades, new techniques have been evolved and utilised. The rectal dialysis ${ }^{4}$ and rectal/ colonic perfusion techniques ${ }^{5}$ have provided important information about the involvement and activity of various inflammatory cells ${ }^{4}{ }^{7}$ but these methods are relatively complicated and are therefore mainly research tools. The advantage of intestinal and colonic perfusion techniques over morphological and immunohistochemical studies of biopsy specimens is that they allow quantitative evaluation of the release of humoral substances and cells in the diseased gut mucosa. ${ }^{58}$ We ourselves have previously developed an intestinal perfusion technique $e^{5}$ but for large clinical studies we have recognised the need for a more simple method that is safe, fast, and inexpensive, but still reliable for assessing inflammation of the gut mucosa.

The aim of this study was to develop and evaluate a new instrument for studying inflammation in the rectal mucosa. We report the design of this instrument, the "mucosal patch technique", and the results obtained in healthy controls and patients with inflammatory bowel diseases (IBD), irritable bowel syndrome (IBS), and coeliac disease. In the present study, we assessed inflammation by measuring inflammatory mediators of neutrophils (myeloperoxidase (MPO) and human neutrophil lipocalin (HNL)) and eosinophils (eosinophilic cationic protein (ECP)).

\section{SUBJECTS AND METHODS \\ Subjects}

Eighty one subjects and patients, 40 men and 41 women, were recruited at the Section of Gastroenterology at the University Hospital of Uppsala. The number of patients in the different diagnostic groups and their sex and age range are given in table 1. Diagnoses of IBD were based on clinical and endoscopic criteria" and $x$ ray of the small intestine. For grading the activity of ulcerative colitis (UC), a four graded endoscopic scale was used where a score of 1 represents normal mucosa and scores 2-4 imply increasing degrees of visible inflammation (score 2 represents slight granularity and/or fragility, score 3 is a mucosa with pronounced granularity and fragility with pus and/or blood in the lumen, and score 4 is a mucosa with pronounced granularity and fragility together with ulcerations). ${ }^{9}{ }^{10}$ Patients with UC had a mean disease duration of 6.5 years (range 1 month to 22 years). Patients were judged to have inactive UC when normal macroscopic findings at endoscopy were combined with normal blood tests and normal bowel movements. Active UC was diagnosed on the basis of clinical symptoms and when the endoscopic score was 2-4. Six of eight patients with inactive UC and 16 of 19 patients with active UC were receiving 5-aminosalicylic acid containing drugs at a dose of

Abbreviations: MPO, myeloperoxidase; $\mathrm{HNL}$, human neutrophil lipocalin; ECP, eosinophil cationic protein; IBS, irritable bowel syndrome; IBD, inflammatory bowel diseases; UC, ulcerative colitis; CC, collagen colitis; CTAB, N-cetyl-N,N,N-trimethyl ammonium bromide 
Table 1 Diagnosis, sex, and ages of the patients and controls included in the study

\begin{tabular}{lccll}
\hline Diagnosis & $\mathbf{n}$ & Sex (F/M) & $\begin{array}{l}\text { Age }(\mathbf{y}) \\
\text { (mean (median)) }\end{array}$ & $\begin{array}{l}\text { Age }(\mathbf{y}) \\
\text { (range) }\end{array}$ \\
\hline Ulcerative colitis active & 19 & $5 / 14$ & $43.4(43,0)$ & $19-79$ \\
Ulcerative colitis inactive & 8 & $3 / 5$ & $47.8(50.0)$ & $26-66$ \\
Collagen colitis & 12 & $10 / 2$ & $52.3(48,5)$ & $33-86$ \\
Irritable bowel disease & 13 & $11 / 2$ & $38.9(36.0)$ & $22-61$ \\
Coeliac disease & 13 & $7 / 6$ & $49.8(55.0)$ & $25-68$ \\
Healthy controls & 16 & $5 / 11$ & $29.1(22.0)$ & $19-58$ \\
\hline
\end{tabular}

0.8-4.0 g/day. Nine patients with active UC were on glucocorticoid treatment at the time of the present investigation. One patient with active UC was receiving azathioprine. Five patients with inactive UC and eight patients with active UC had total colitis. The remaining patients suffered from distal colitis of varying extents. Two patients in the active and one patient in the inactive group had only proctitis. Patients with collagen colitis (CC) were diagnosed by clinical symptoms and colonic biopsy. IBS patients fulfilled the Rome II criteria. ${ }^{11}$ Eight of these were diarrhoea predominant and five had an alternating type of IBS. Patients with coeliac disease were all diagnosed on the basis of two duodenal biopsies showing regression or normalisation of the duodenal mucosa after a gluten free diet. In patients with coeliac disease who still had symptoms on a gluten free diet (diarrhoea, abdominal pain, blood in stools, urgency, etc) an investigation with fibresigmoidoscopy was performed and biopsies taken to exclude microscopic colitis. One patient with coeliac disease was excluded because of biopsy verified microscopic colitis.

Patients with haemorrhoids, rectal fistulas, and anorectal prolapses were excluded, as were patients with severe systemic illness, ongoing viral or bacterial infections, or known latex allergy, and patients operated on in the last six months.

Sixteen control subjects were recruited among healthy students, nurses, and their relatives. All controls included in the study had no gastrointestinal history and no symptoms of any kind of illnesses in the last two months. A simple cold was accepted if it had resolved a week before measurements. All had normal results following a wide number of blood screening tests before and at the time of the study.

All participants of the study gave informed consent. The ethics committee of the Medical Faculty, Uppsala University, approved the study.

\section{Subject preparation}

Patients were prepared in three different ways before being tested with the rectal mucosal patch technique:

(1) 20 patients were prepared as for routine colonoscopy (that is, they drank $4 \mathrm{l}$ of an isotonic fluid-Laxabon; Hässle, Lund, Sweden);

(2) 51 patients were prepared as for routine fibresigmoidoscopy, with a non-irritative hyperosmolar sorbitol rectal enema 120-240 ml (Klyx; Ferring, Malmö, Sweden) one hour before the test. Four of these patients also underwent the test after Laxabon preparation; and

(3) 10 patients performed the test without prior preparation because they suffered from active UC.

\section{Design and positioning of the instrument}

The instrument used for the mucosal patch technique is a plastic catheter with a silicon balloon at the end of the catheter, with two or three patches attached to the balloon (fig 1). The patches are made of highly absorptive cellulose material (Pharmacia Diagnostics AB, Uppsala, Sweden). The tip of the instrument is cone shaped and therefore easy to introduce without endoscopic guidance.

When the instrument is positioned in the rectum, the balloon is inflated with air $(60-80 \mathrm{ml})$, allowing the patches to be in contact with the mucosa (fig $1 \mathrm{~B}$, fig 2 ). Two latex shields protect the patches during introduction of the instrument into the rectum and during its retraction (fig lA).

If the patient had a strong feeling of distension or pain that did not disappear after 2-3 minutes, the volume of air was reduced by $5 \mathrm{ml}$ at a time until tolerance was reached. A minimum of $50 \mathrm{ml}$ of air was left in the balloon to ensure that the patches remained adherent to the mucosa. The balloon was kept inflated for 20 minutes in all subjects, but 15 subjects also agreed to do three more tests for 15, 10, and 5 minutes. The aim was to determine the time dependency of absorption of the measured substances to the patches. After 20 minutes the balloon was deflated and the catheter removed. The patches were cut off and immediately placed in $2 \mathrm{ml}$ of $0.3 \%$ CTAB ( $\mathrm{N}$-cetyl-N,N,N-trimethyl ammonium bromide; E Merck, Darmstadt, Germany) solution to extract the contents. At each measurement a blood sample was taken. The aim was to evaluate systemic inflammatory activity for comparison with the measurements of the patches.

Any discomfort, reactions, and other symptoms during the test procedure were recorded, as was the presence of blood or faeces on the patches.

\section{Analytical measurements}

Each patch was collected and analysed separately; therefore, there were three sets of measurements for each test procedure. The patches were kept in $0.3 \%$ CTAB solution for extraction for one hour. After completion of this step, the extraction solution was squeezed out of the patches, centrifuged, and then frozen at $-70^{\circ} \mathrm{C}$ until analysed. Blood samples were centrifuged and serum frozen at $-70^{\circ} \mathrm{C}$ until analysed.

Three endogenous substances representing aspects of the inflammatory process were measured in the solution or serum: HNL and MPO as markers of neutrophil activity, and ECP as a marker of eosinophil granular release. Cellular specificities have recently been reviewed ${ }^{12}$ and the specificity for HNL in the colon has been described. ${ }^{13}$ Duplicate samples were analysed using radioimmunoassays for measuring concentrations of MPO, ECP, and HNL. ${ }^{12}{ }^{14}$

Rectal biopsies were taken around the time of the present investigation (on average \pm 1.8 days) in nine patients with active UC, five with inactive UC, and in 10 patients with IBS. In order to elucidate the link between the measured mediators and rectal histology, a pathologist examined these biopsies without knowledge of the endoscopic activity score or the results of the mediator measurements. Inflammatory activity was assessed using a slightly modified scoring system that has been validated for UC..$^{15}$ 
A

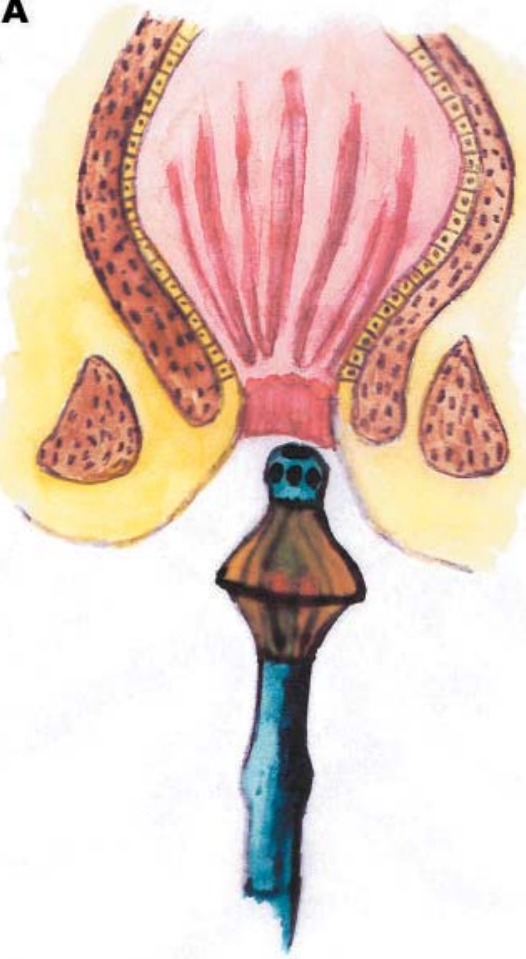

B

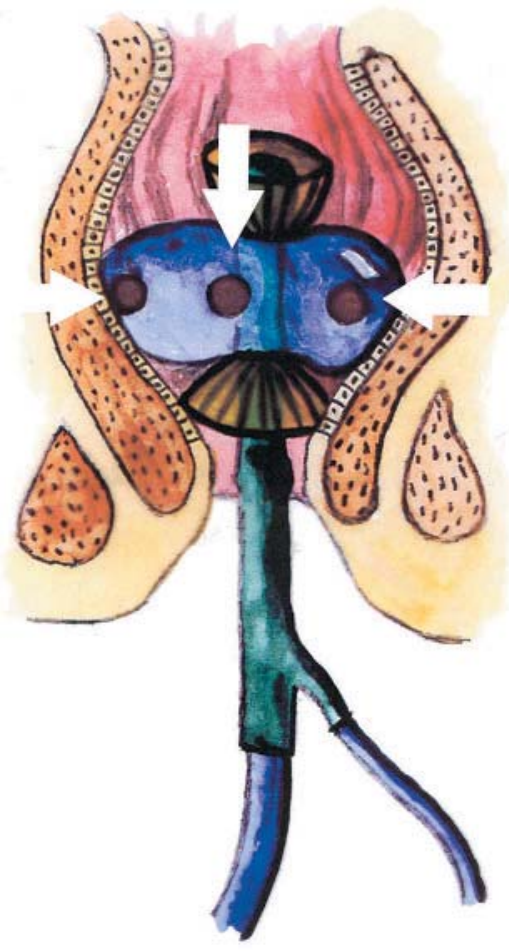

Figure 1 Schematic drawing of the instrument used for the mucosal patch technique for measurement of the inflammatory conditions of the rectal mucosa. (A) The instrument with a noninflated balloon and with the patches protected by shields. (B) The instrument in position with the inflated balloon in the rectal ampulla, and with the patches, indicated by arrows, in contact with the mucosa.
Seven parameters were judged and scored separately, where score " 0 " corresponds to no inflammatory activity (normal histology) and score " 3 " to maximal inflammatory activity or destruction observed. The parameters were: structural (architectural) changes, chronic inflammatory infiltrate, numbers of eosinophilic and neutrophilic granulocytes within the lamina propria, crypt destruction, and erosion or ulceration (see table 4 ).

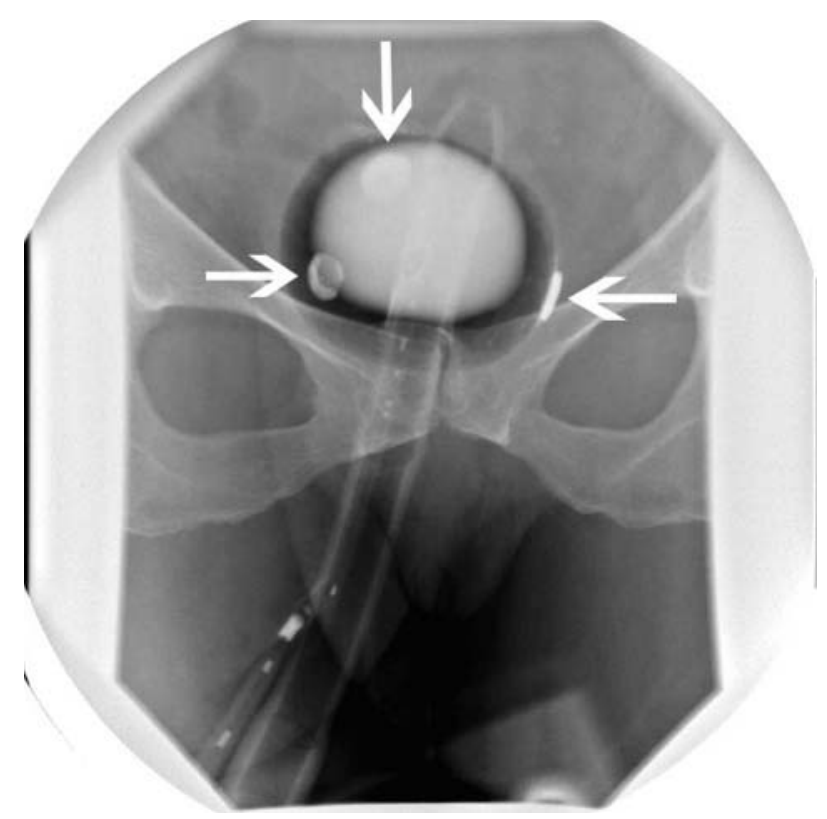

Figure 2 Radiographic appearance of the instrument with an inflated balloon in the rectal ampulla. Arrows indicate the patches in contact with the mucosa.
The last parameter, "erosion or ulceration", had a five point score in the original article. ${ }^{15}$ In the present study, the judgement of "recovering epithelium" was removed thus leaving a scoring system "from 0 to 3 ", ranging from no erosion to ulceration.

\section{Calculations}

The geometric mean value of the measurements of three patches from each individual was calculated. Mean and (SEM) of patient and control groups are presented in the figures, tables, and text.

The Mann-Whitney U, Friedman ANOVA, and Spearman's rank correlation tests were used for statistical analyses. The statistical calculations were performed on a personal computer using the statistical package Statistica (Statsoft Inc., Tulsa, Oklahoma, USA).

\section{RESULTS}

\section{Compliance, tolerance, and safety}

Almost all of the subjects tolerated the test procedure well. Four patients experienced discomfort or pain when the instrument was withdrawn. Six patients complained of a feeling of distension during the rectal procedure; three patients, suffering from UC with grade 4 inflammation, experienced pronounced discomfort. However, they completed the examination after reduction of inflation of air into the balloon. Many patients had a short transient feeling of urgency at the beginning of the test. Twenty two controls and patients underwent colonoscopy 0.5-3 hours after completion of the rectal test, and no signs of trauma to the mucosa were observed.

\section{Extraction from the patches}

In vitro experiments with various $\mathrm{CTAB}$ concentrations were performed in order to test for the optimal procedure of extraction of the patches. The $0.3 \%$ CTAB solution proved to 
Table 2 Time dependency for absorption of granulocyte granule constituents during rectal mucosal measurements

\begin{tabular}{|c|c|c|c|c|c|c|c|c|c|c|c|c|}
\hline \multirow[b]{2}{*}{ Group } & \multicolumn{4}{|c|}{ MPO ( $\mu \mathrm{g} / \mathrm{l})$} & \multicolumn{4}{|c|}{ HNL $(\mu \mathrm{g} / \mathrm{l})$} & \multicolumn{4}{|c|}{ ECP $(\mu \mathrm{g} / \mathrm{l})$} \\
\hline & $5 \mathrm{~min}$ & $10 \mathrm{~min}$ & $15 \min$ & $20 \mathrm{~min}$ & $5 \mathrm{~min}$ & $10 \mathrm{~min}$ & $15 \min$ & $20 \mathrm{~min}$ & $5 \mathrm{~min}$ & $10 \min$ & $15 \mathrm{~min}$ & $20 \mathrm{~min}$ \\
\hline $\begin{array}{l}\text { Low } \\
(n=5) \\
\text { Medium } \\
(n=5) \\
\text { High } \\
(n=5)\end{array}$ & $\begin{array}{l}15.0 \\
(3.4) \\
101.2 \\
(34.1) \\
4152.2 \\
(1891.1)\end{array}$ & $\begin{array}{l}14.8 \\
(3.8) \\
119.5 \\
(41.0) \\
3872.5 \\
(1735.9)\end{array}$ & $\begin{array}{l}13.9 \\
(2.02) \\
110.0 \\
(28.3) \\
4587.4 \\
(2107.2)\end{array}$ & $\begin{array}{l}16.4 \\
(2.8) \\
266.5 \\
(98.3) \\
3874.2 \\
(1661.2)\end{array}$ & $\begin{array}{l}13.4 \\
(1.6) \\
34.3 \\
(8.1) \\
901.2 \\
(296.2)\end{array}$ & $\begin{array}{l}15.1 \\
(0.8) \\
39.0 \\
(11.0) \\
950.1 \\
(239.4)\end{array}$ & $\begin{array}{l}17.0 \\
(1.2) \\
35.1 \\
(11.0) \\
1103.9 \\
(362.8)\end{array}$ & $\begin{array}{l}18.1 \\
(3.7) \\
79.6 \\
(29.7) \\
1106.2 \\
(402.0)\end{array}$ & $\begin{array}{l}21.4 \\
(4.2) \\
43.1 \\
(28.0) \\
866.4 \\
(317.7)\end{array}$ & $\begin{array}{l}23.1 \\
(6.4) \\
56.0 \\
(37.2) \\
787.6 \\
(316.0)\end{array}$ & $\begin{array}{l}37.9 \\
(17.5) \\
62.9 \\
(45.6) \\
899.7 \\
(321.3)\end{array}$ & $\begin{array}{l}56.0 \\
(27.4) \\
55.8 \\
(24.6) \\
803.7 \\
(295.8)\end{array}$ \\
\hline
\end{tabular}

Concentrations of myeloperoxidase (MPO), human neutrophil lipocalin (HNL), and eosinophil cationic protein (ECP) after 5, 10, 15, and 20 minutes in groups with low, medium, and high inflammatory activity.

Values are mean (SEM).

give acceptable recovery of MPO and ECP added in vitro to the patches; the recovery of these substances was $>90 \%$.

\section{Influence of subject preparation}

The type of gut preparation (hyperosmolar sorbitol rectal enema or oral laxative isotonic solution) had no significant influence on the recovered amounts of MPO or ECP in tested patients with normal endoscopic findings $(n=26)$ and healthy controls $(n=16)$.

\section{Intraindividual variation}

In 11 patients with coeliac disease and three healthy controls, two separate measurements were performed on two occasions with an interval of at least one week. Mean MPO concentration was $43.2(13.1) \mu \mathrm{g} / \mathrm{l}$ on the first test and 54.6 (14.8) $\mu \mathrm{g} / \mathrm{l}$ on the second test $(\mathrm{p}=0.6)$. Corresponding ECP values were $29.2(7.0) \mu \mathrm{g} / \mathrm{l}$ and $30.5(7.4) \mu \mathrm{g} / \mathrm{l}(\mathrm{p}=0.79)$, respectively.

\section{Interpatch variation}

The mean coefficient of variation (between results obtained from duplicate patches of the same instrument was, on average, $38.1 \%(n=84)$.

\section{Time dependency of absorption}

Fifteen patients were tested with the instrument positioned in the rectum for various lengths of time. Subjects were divided into three groups on the basis of the degree of inflammatory activity, defined by rectal recovery of the granulocyte markers: those with high $(n=5)$, medium $(n=5)$, and low levels $(n=5)$ of inflammatory markers. Results are presented in table 2. Time dependency was not critical for the groups with high and low inflammatory activity but in the medium group there was a tendency to higher values with time; this difference was not statistically significant $(\mathrm{p}=0.11)$.

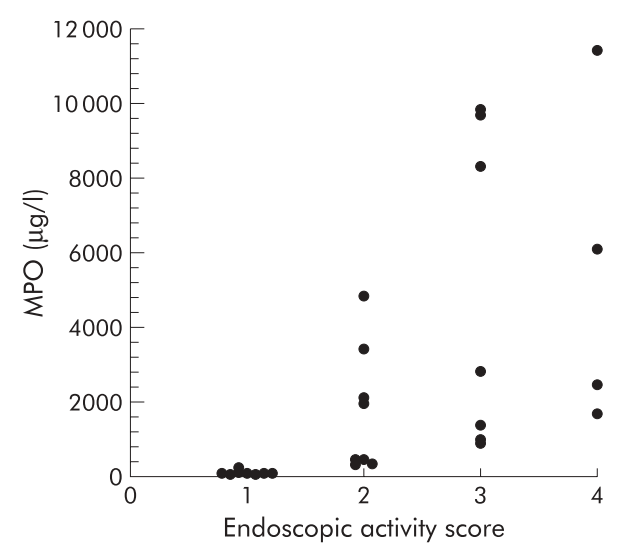

Figure 3 Correlation between myeloperoxidase (MPO) levels and endoscopic disease activity score in ulcerative colitis patients $(n=27)$. A positive correlation (Spearman $R=0.81, p<0.001$ ) was found.

\section{Patient and control measurements \\ Mucosal measurements}

Table 3 shows the mean recovered mucosal amounts of MPO, HNL, and ECP in the patient and control groups. An apparent covariation was seen between MPO and HNL ( $r=0.94$ for all individuals). Significantly higher concentrations of all three variables were observed in patients with active UC compared with those with UC in remission. A positive correlation (Spearman $r=0.81, \mathrm{p}<0.001$ ) was found between endoscopic disease activity score and MPO concentration in UC patients (fig 3). Endoscopic findings were also significantly correlated to HNL (Spearman $r=0.78, \mathrm{p}<0.001$ ) and ECP concentrations (Spearman $r=0.61, \mathrm{p}<0.001$ ). Other patient groups all had significantly lower concentrations of the measured substances compared with patients with active UC (table 3).

Table 3 Rectal mucosal concentrations of myeloperoxidase (MPO), eosinophil cationic protein (ECP), and human neutrophil lipocalin (HNL) in patients with ulcerative colitis (UC), coeliac disease, and irritable bowel syndrome (IBS), and in healthy controls

\begin{tabular}{|c|c|c|c|c|c|c|}
\hline & MPO ( $\mu \mathrm{g} / \mathrm{l})$ & S-MPO ( $\mu \mathrm{g} / \mathrm{l})$ & $\mathrm{ECP}(\mu \mathrm{g} / \mathrm{l})$ & S-ECP $(\mu \mathrm{g} / \mathrm{l})$ & HNL $(\mu \mathrm{g} / \mathrm{l})$ & S-HNL ( $\mu \mathrm{g} / \mathrm{l})$ \\
\hline Active UC $(n=19)$ & $3205.8(774.8)^{\star * \star}$ & $357.4(24.8)$ & $374.6(100.8)^{\star * *}$ & $10.9(1.4)$ & $677.0(171.3)^{* * *}$ & $146.4(16.3)^{*}(n=18)$ \\
\hline Inactive UC $(n=8)$ & $59.6(22.2)^{\star *}$ & $233.0(56.4)$ & $31.3(17.3)$ & $13.1(4.0)$ & $49.7(8.7)^{\star \star \star}$ & $101.8(21.1)$ \\
\hline Collagen colitis $(n=12)$ & $53.6(21.9)^{*}$ & $298.9(12.5)$ & $81.9(24,2)$ & $11.5(1.4)$ & $34.7(6.7)^{\star *}$ & $99.3(6.4)$ \\
\hline IBS $(n=13)$ & $44.6(9.6)^{* *}$ & $253.0(25.1)$ & $25.7(4.7)$ & $13.3(1.8)$ & $33.7(5.1)^{\star * *}$ & $97.8(11.0)$ \\
\hline Coeliac disease $(n=13)$ & $74.0(22.4)^{\star *}$ & $209.0(26.5)$ & $30.5(27.5)$ & $11.7(2.5)$ & $28.7(5.4)^{* *}$ & $96.1(6.7)$ \\
\hline Healthy controls $(n=16)$ & $11.7(2.6)$ & $276.7(35.5)$ & $32.7(7.2)$ & $12.0(1.4)$ & $12.9(1.6)$ & $100.5(8.7)$ \\
\hline
\end{tabular}




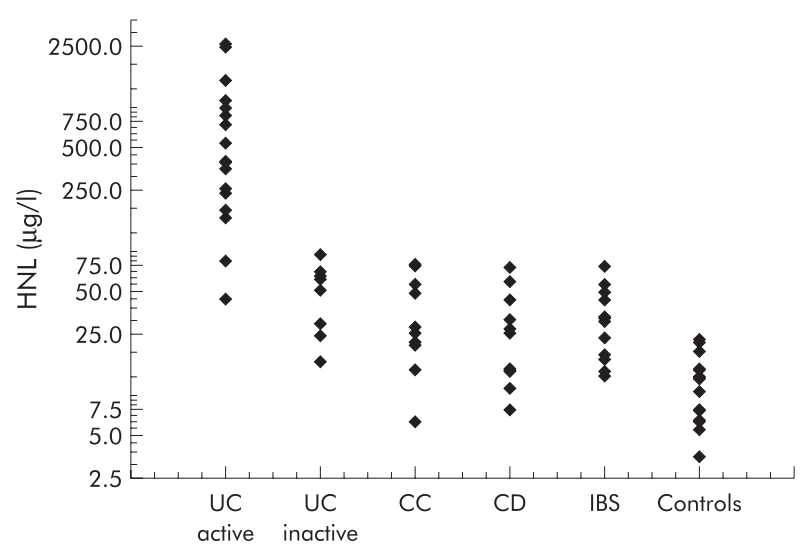

Figure 4 Individual human neutrophil lipocalin (HNL) concentrations recovered from the rectal mucosa of patients with active and inactive ulcerative colitis (UC), collagen colitis (CC), coeliac disease (CD) irritable bowel syndrome (IBS), and healthy controls.

Patients with coeliac disease, CC, and IBS had significantly higher levels of MPO and HNL than healthy controls (table 3 ). In fig 4, individual results of HNL in the different patient groups and controls are shown

\section{Mucosal measurements and histological assessment of inflammation}

Measured inflammatory mediators were related to different histological grades of disease severity in those patients who had biopsies taken near the time of the mucosal measurements. The findings presented in table 4 show that MPO, HNL, and ECP values correlated with the mucosal structural changes and the increase in chronic inflammatory infiltrate. The presence of neutrophils in the lamina propria and in the epithelium correlated with the neutrophil markers MPO and HNL, and the presence of eosinophils in the lamina propria correlated with the eosinophil marker ECP. Histological findings in patients with IBS were characterised by no increase or a mild but unequivocal increase in eosinophils in the lamina propia. Correlations observed between the number of mucosal neutrophils/eosinophils and neutrophil/ eosinophil markers in patients with UC remained when calculations were performed in all patients.

\section{Serum measurements}

Serum concentrations of HNL in patients with active UC were significantly higher than those in patients with IBS, coeliac disease, and healthy controls (table $3, \mathrm{p}<0.05$ ). However, differences in serum values were relatively marginal compared with the observed differences in mucosal measurements.

\section{DISCUSSION}

In this study we have presented a new mucosal mapping technique for measuring markers of events in the colorectal mucosa and shown that it is simple to use, safe, and very reliable, and that patient compliance is good. These characteristics demonstrate the potential of the system as a practical clinical tool for evaluating inflammatory markers in many settings.

Compared with other methods, ${ }^{45816} 17$ the present system has several advantages. In our experience it is technically simpler to perform and more comfortable for patients than rectal dialysis or rectal perfusion techniques. ${ }^{45}$ The size of the molecule to be evaluated is not critical, as it is in rectal dialysis. It does not require an endoscopic procedure, as in rectal perfusion and in the filter technique described by Hendel and colleagues, ${ }^{16}$ but it can be easily combined with endoscopy when required. The perfusion technique has been described as the golden standard for measurement of inflammatory mediators in the gut but its use is limited to research studies because of its complexity. Perfusion of the gut also dilutes the amounts of inflammatory substances, which means that low levels in non-inflammatory conditions and healthy controls are below the detection limit. ${ }^{18}$ Our new technique is very sensitive and permits detection of inflammatory cells even in normal mucosa by virtue of the extreme absorption capacity of the cellulose material used for the test procedure. Studies of the time needed for the cellulose material to absorb inflammatory substances from the mucosa indicated that after less than 20 minutes, equilibrium between the mucosa and cellulose was reached. For practical clinical purposes it seems quite acceptable to reduce the time of contact between the cellulose material and the mucosa to approximately 10 minutes. Studies in vitro of the highly absorptive cellulose patches demonstrated acceptable recovery of absorbed inflammatory substances with the extraction procedure used.

Preparation of the gut is essential in situations where the release of inflammatory substances to the gut lumen is measured as faecal contamination may influence the results. ${ }^{19-21}$ It has also been reported that a hyperosmolar solution may affect the mucosa if osmolarity is more than $1500 \mathrm{mosmol} / \mathrm{l}{ }^{22}$ The enema we used has an osmolarity of $1686 \mathrm{mosmol} / \mathrm{l}$. However, preparation with an isotonic fluid and a hyperosmolar enema gave identical results in the present system and neither method induced any apparent inflammatory reaction in the rectal mucosa; these findings are in accordance with the results obtained with gut preparation prior to the perfusion technique. ${ }^{7}$ In this context it should be mentioned that repeated exposure of the rectal mucosa to a hyperosmolar milieu (2-3 enemas within 24 hours) induced an inflammatory reaction in some healthy controls (unpublished observations).

One advantage of the perfusion technique for assessing the inflammatory activity of the rectal mucosa is that results obtained by perfusion of a defined gut segment give an

Table 4 Correlation coefficients between mucosal inflammatory mediators and histological grades of disease activity in patients with ulcerative colitis

\begin{tabular}{llll}
\hline & MPO & HNL & ECP \\
\hline Structural (architectural change) & $0.75^{* *}$ & $0.75^{* *}$ & $0.80^{* *}$ \\
Chronic inflammatory infiltrate & $0.77^{* *}$ & $0.79^{* *}$ & $0.76^{* *}$ \\
Lamina propria eosinophils & 0.46 & 0.48 & $0.64^{*}$ \\
Lamina propria neutrophils & $0.75^{* *}$ & $0.77^{* *}$ & $0.61^{*}$ \\
Neutrophils in epithelium & $0.55^{*}$ & $0.59^{*}$ & 0.38 \\
Crypt destruction & 0.41 & 0.45 & 0.22 \\
Erosion or ulceration & 0.49 & $0.57^{*}$ & 0.40 \\
\hline MPO, myeloperoxidase; HNL, human neutrophil lipocalin; ECP, eosinophil cationic protein. \\
${ }^{*} \mathrm{p}<0.05$ and ** $\mathrm{p}<0.01$ according to Spearman rank correlation.
\end{tabular}


average value for mucosal inflammation. ${ }^{58}$ Patchiness of mucosal inflammation in UC has previously been emphasised. ${ }^{23}{ }^{24}$ In an attempt to compensate for possible regional differences in the inflammatory status of the rectal mucosa, we performed three measurements at different rectal sites. The average intraindividual variation of the three rectal measurements was $38 \%$, thus illustrating the expected variability in a mucosal inflammatory process. The difference in the mean of such measurements performed at different times was not significant. Thus reproducibility was quite acceptable with this process, which implies that longitudinal studies of the condition of the mucosa can be made in individual patients.

Another strength of the present technique is its ability to detect the activity of neutrophil and eosinophil granulocytes in apparently healthy rectal mucosa. With the intestinal perfusion technique we have not previously been able to define the normal activity of inflammatory cells in the gut mucosa. ${ }^{78} 19$ The advantage of a high sensitivity method was obvious in the present study as subclinical inflammation in the rectal mucosa was detected in patients with IBS. Although IBS is not regarded as an inflammatory disease, its inflammatory basis has been discussed, and non-specific mild inflammation in the colonic mucosa is often seen histologically in IBS patients. ${ }^{25-27}$ It has been proposed that such mucosal inflammatory findings might be explained by undiagnosed coeliac disease or postinfective IBS. ${ }^{27} 28$ However, such possible explanations are not relevant to our findings in IBS as only two of our IBS patients had a history of IBS secondary to a gastrointestinal infection which occurred more than three years before the present investigation. Furthermore, all of our IBS patients had normal duodenal biopsies and were negative for serological markers of coeliac disease.

Previous examinations of colonoscopic biopsy specimens from patients meeting the Rome criteria for a clinical diagnosis of IBS have given conflicting results. Chadwick and colleagues ${ }^{27}$ promoted the hypothesis that in at least a subset of patients with IBS low grade inflammation with increased numbers of neutrophils and mast cells might play a pathophysiological role. Others ${ }^{29}$ have reported, in this disease, increased numbers of mast cells in proximal colonic mucosa but no increase in these cells or neutrophils in the rectum. As overt colonic inflammation precludes a diagnosis of IBS, the need for more sensitive markers of low grade inflammation has been stressed. ${ }^{29}$ Counting single inflammatory cell subtypes per high power field has the potential to be more sensitive compared with our histological method (validated for UC to assess the degree of mucosal inflammation). Conventional histological examination of biopsies identifies the number of inflammatory cells but gives no information on the degree of granulocyte activation. Increased release of granular constituents is one sign of granulocyte activation. Our finding of increased mucosal amounts of neutrophil granular proteins (MPO and HNL) in spite of no apparent increase in neutrophils might therefore reflect low grade inflammation. This interpretation is supported by the finding that IBS patients have significantly increased amounts of faecal calprotectin, a neutrophil derived protein. ${ }^{30}$ Faecal protein amounts may originate from any part of an inflamed gut. We cannot fully exclude the fact that mediators absorbed in the rectum by the patches to some extent may originate from other sites of an inflamed colon. However, our preinvestigation preparation of patients and local application of the absorbent patches reduce such influences. The correlation between measured inflammatory mediators and endoscopic and histological findings in UC indicate that our technique reflects mainly a local inflammatory process.
The finding of subclinical rectal inflammatory activity in diet treated coeliac disease was unexpected but previous reports of proctitis in this disease deserve attention. ${ }^{31}{ }^{32}$ Cellier et al found that a gluten free diet induced regression but not normalisation of $\mathrm{T}$ cell activation in the rectal mucosa of patients with coeliac disease. ${ }^{32}$ Their report together with our present results suggests a non-gluten related increase in the inflammatory propensity of the gut mucosa in this disease. It is well known that gluten induces not only a lymphocytic response but also increased mucosal neutrophil infiltration. $^{33}$

Patients with active UC showed, on average, more than 300 -fold increase in the inflammatory activity of the rectal mucosa, as judged by recovered amounts of MPO. The involvement of eosinophils in this disease was also clear from the several-fold increase in the recovery of ECP, and our findings are in accordance with our previous report using a perfusion technique. ${ }^{7}$ Patients with inactive UC showed normalisation of eosinophil activity of the mucosa but neutrophil activity remained increased compared with that of healthy controls but of the same magnitude as observed in IBS and coeliac disease. The potential of this sensitive method for assessment of inflammatory activity was also illustrated by the significantly increased recovery of neutrophil granule constituents in our patients with CC. In a minority of these patients, mucosal recovery of ECP was highly increased in accordance with recently published data from a study using rectal perfusion. ${ }^{34}$

In summary, we have described a new principle for assessing biological events in the colonic mucosa and have applied it in studies of the rectal mucosa in health and in patients with various inflammatory and non-inflammatory bowel diseases. We conclude that this new technique is rapid, simple, safe, and highly sensitive, and produces reliable and reproducible data. The results obtained indicate that it might be suitable not only for practical clinical use but also for research studies of gut pathophysiology.

\section{ACKNOWLEDGEMENTS}

We acknowledge the technical assistance of Sneh Ajuha, Kerstin Lindblad, and Asa Lidman. This work was supported by the medical faculty of the University of Uppsala, Sweden, Pharmacia Diagnostics $\mathrm{AB}$, Uppsala, Sweden, Alimenta Diagnostics AB, Uppsala, Sweden, and by the Vardal Foundation-the Swedish Foundation for Health Care Sciences and Allergy Research.

\section{Authors' affiliations \\ G Kristiánsson, L Lööf*, Section of Gastroenterology, Department of Medical Sciences, University of Uppsala, Uppsala, Sweden \\ P Venge, Laboratory for Inflammation Research, University of Uppsala, Uppsala, Sweden \\ A Wanders, Department of Pathology, University of Uppsala, Uppsala, Sweden \\ R Hällgren, Department of Rheumatology, University of Uppsala, Uppsala, Sweden}

*Current address: Centre for Clinical Research, Central Hospital, Västerås, Sweden

\section{REFERENCES}

1 Heatley RV, James PD. Eosinophils in the rectal mucosa. A simple method of predicting the outcome of ulcerative proctocolitis? Gut 1979;20:787-91.

2 Allison MC, Poulter LW, Dhillon AP, et al. Immunohistological studies of surface antigen on colonic lymphoid cells in normal and inflamed mucosa. Comparison of follicular and lamina propria lymphocytes. Gastroenterology 1990;99:421-30

3 MacDonald TT, Horton MA, Choy MY, et al. Increased expression of laminin/ collagen receptor (VLA-1) on epithelium of inflamed human intestine. J Clin Pathol 1990;43:313-15.

4 Nielsen $\mathrm{OH}$, Gionchetti $P$, Ainsworth $M$, et al. Rectal dialysate and fecal concentrations of neutrophil gelatinase-associated lipocalin, interleukin-8, and 
fumor necrosis factor-alpha in ulcerative colitis. Am J Gastroenterol 1999; $94: 2923-8$.

5 Raab Y, Hällgren R, Knutson $L$, et al. A technique for segmental rectal and colonic perfusion in humans. Am J Gastroenterol 1992;87:1453-9.

6 Raab Y, Gerdin B, Ahlstedt S, et al. Neutrophil mucosal involvement is accompanied by enhanced local production of interleukin-8 in ulcerative colitis. Gut 1993;34:1203-6.

7 Raab Y, Fredens K, Gerdin B, et al. Eosinophil activation in ulcerative colitis: studies on mucosal release and localization of eosinophil granule constituents. Dig Dis Sci 1998;43:1061-70.

8 Knutson L, Odlind B, Hällgren R. A new technique for segmental jejunal perfusion in man. Am J Gastroenterol 1989;84:1278-84.

9 Binder V. A comparison between clinical state, macroscopic and microscopic appearances of rectal mucosa, and cytologic picture of mucosal exudate in ulcerative colitis. Scand J Gastroenterol 1970:5:627-32.

10 Baron JH CA, Lennard Jones JE. Variation between describing mucosal appearance in proctocolitis. BMJ 1964;1:89-92.

11 Thompson WG, Longstreth GF, Drossman DA, et al. Functional bowel disorders and functional abdominal pain. Gut 1999:45(suppl 2):|143-7.

12 Venge P. Monitoring the allergic inflammation. Allergy 2004;59:26-32.

13 Carlson M, Raab Y, Seveus L, et al. Human neutrophil lipocalin is a unique marker of neutrophil inflammation in ulcerative colitis and proctitis. Gut 2002;50:501-6.

14 Xu SY, Petersson CG, Carlson M, et al. The development of an assay for human neutrophil lipocalin (HNL)- to be used as a specific marker of neutrophil activity in vivo and vitro. J Immunol Methods 1994;171:245-52.

15 Geboes K, Riddell R, Öst $\AA$, et al. A reproducible grading scale for histological assessment of inflammation in ulcerative colitis. Gut 2000:47:404-9.

16 Hendel J, Nielsen $\mathrm{OH}$, Madsen S, et al. A simple filter-paper technique allows detection of mucosal cytokine levels in vivo in ulcerative colitis. Interleukin-1 and interleukin-1-receptor antagonist. Dig Dis Sci 1996;41:1775-9.

17 Saitoh H, Takagaki K, Nakamura T, et al. Characterization of mucin in wholegut lavage fluid obtained from patients with inflammatory bowel disease. Dig Dis Sci 1996;41:1768-74.

18 Sangfelt $\mathbf{P}$, Carlson $M$, Thörn $M$, et al. Neutrophil and eosinophil granule proteins as markers of response to local prednisolone treatment in distal ulcerative colitis and proctitis. Am J Gastroenterol 2001;96:1085-90.

19 Hällgren R, Colombel JF, Dahl R, et al. Neutrophil and eosinophil involvement of the small bowel in patients with celiac disease and Crohn's disease: studies on the secretion rate and immunohistochemical localization of granulocyte granule constituents. Am J Med 1989;86:56-64.
20 Peterson CG, Eklund $E$, Taha $Y$, et al. A new method for the quantification of neutrophil and eosinophil cationic proteins in feces: establishment of normal levels and clinical application in patients with inflammatory bowel disease. Am J Gastroenterol 2002;97:1755-62.

21 Bischoff SC, Grabowsky J, Manns MP. Quantification of inflammatory mediators in stool samples of patients with inflammatory bowel disorders and controls. Dig Dis Sci 1997;42:394-403.

22 Wheeler PG, Menzies IS, Creamer B. Effect of hyperosmolar stimuli and coeliac disease on the permeability of the human gastrointestinal tract. Clin Sci Mol Med 1978:54:495-501.

23 Bernstein CN, Shanahan F, Anton PA, et al. Patchiness of mucosal inflammation in treated ulcerative colitis: a prospective study. Gastrointest Endosc 1995;42:232-7.

24 Kleer CG Appelman HD. Ulcerative colitis: patterns of involvement in colorectal biopsies and changes with time. Am J Surg Pathol 1998;22:983-9.

25 Khan I CS. Is there an inflammatory basis for a subset of patients presenting with diarrhoea in the irritable bowel syndrome. Gastroenterology 1994; 106:A523.

26 Lee E, Schiller LR, Fordtran JS. Quantification of colonic lamina propria cells by means of a morphometric point-counting method. Gastroenterology 1988;94:409-18.

27 Chadwick VS, Chen W, Shu D, et al. Activation of the mucosal immune system in irritable bowel syndrome. Gastroenterology 2002;122:1778-83.

28 Spiller RC, Jenkins D, Thornley JP, et al. Increased rectal mucosal enteroendocrine cells, T lymphocytes, and increased gut permeability following acute Campylobacter enteritis and in post-dysenteric irritable bowel syndrome. Gut 2000;47:804-11

29 O'Sullivan M. Clayton N, Breslin NP, et al. Increased mast cells in the irritable bowel syndrome. Neurogastroenterol Motil 2000;12:449-57.

30 Tibble J, Teahon K, Thiodleifsson B, et al. A simple method for assessing intestinal inflammation in Crohn's disease. Gut 2000;47:506-13.

31 Breen EG, Coughlan G, Connolly CE, et al. Coeliac proctitis. Scand J Gastroenterol 1987:22:471-7.

32 Cellier C, Cervoni JP, Patey N, et al. Gluten-free diet induces regression of Tcell activation in the rectal mucosa of patients with celiac disease. Am J Gastroenterol 1998;93:1527-30.

33 Ensari A, Ager A, Marsh MN, et al. Time-course of adhesion molecule expression in rectal mucosa of gluten-sensitive subjects after gluten challenge. Clin Exp Immunol 1993;92:303-7.

34 Taha Y, Carlson M, Thörn M, et al. Evidence of local eosinophil activation and altered mucosal permeability in collagenous colitis. Dig Dis Sci 2001;46:888-97.

\section{EDITOR'S QUIZ: GI SNAPSHOTS}

\section{Answer}

From question on page 1793

The radiograph (fig 1) shows displacement of the liver and compression of intestinal loops by a tension pneumoperitoneum. After insertion of a 14 gauge angiocatheter through the abdominal wall, the abdomen deflated. Immediate laparotomy revealed a lateral duodenal perforation and intestinal infarction from the proximal jejunum to the proximal ileum. Endoscopic retrograde cholangiography (ERC) was difficult because of duodenal obstruction due to Waldenströem's macroglobulinaemia (WM) involvement of the duodenum. Insufflated air probably caused duodenal pneumatosis and finally perforation. The air escaped into the retroperitoneum, peritoneum, and subcutaneous tissue. An increase in intra-abdominal pressure together with enlarged para-aortal lymph nodes resulted in compression of the vena cava inferior and splanchnic vessels, cardiac low output syndrome, and respiratory dysfunction. WM associated hyperviscosity because of circulating IgM paraprotein may have resulted in impaired microcirculation with segmental bowel infarction in a patient without symptoms of previous intestinal ischaemia. Despite rapid surgical intervention the patient died from refractory acidosis and gastrointestinal bleeding.

doi: $10.1136 /$ gut.2003.031211 